\title{
Cellular Fatty Acid Composition Comparisons of Haemophilus equigenitalis and Moraxella Species
}

\author{
C. SUGIMOTO,* E. MIYAGAWA, M. NAKAZAWA, K. MITANI, AND Y. ISAYAMA
}

First Research Division, National Institute of Animal Health, Yatabe, Tsukuba, Ibaraki 305, Japan

\begin{abstract}
The cellular fatty acid compositions of Haemophilus equigenitalis and some other species were compared. The cellular fatty acid composition of $H$. equigenitalis was very similar to the cellular fatty acid compositions of Moraxella species. When the double bond positions in monounsaturated fatty acids were determined by a gas chromatography-mass spectrometry analysis of ditrimethylsilyloxy derivatives, Moraxella species were divided into two groups, the oleic acid group and the cis-vaccenic acid group. The former included eight species (Moraxella atlantae, Moraxella bovis, Moraxella caviae, Moraxella equi, Moraxella lacunata, Moraxella nonliquefaciens, Moraxella osloensis, and Moraxella phenylpyruvica), and the latter included Moraxella catarrhalis, Moraxella ovis, and Moraxella urethralis. $H$. equigenitalis was closely related to the cis-vaccenic acid group.
\end{abstract}

The taxonomic position of Haemophilus equigenitalis, the causative agent of contagious equine metritis, is still unclear because this organism does not require X-or V-factors $(7,29$, 31). Recently, we studied the cellular fatty acid composition of $\boldsymbol{H}$. equigenitalis and found that it is characterized by large amounts of $18: 1$ and 16:0 acids (30). The cellular fatty acid composition of this species is grossly similar to the cellular fatty acid compositions of Moraxella species $(5,9,14,15,18,27)$. In addition, $H$. equigenitalis is catalase and oxidase positive, asaccharolytic, and very unreactive in the conventional biochemical tests (31) and thus is phenotypically similar to species of the genus Moraxella (12). However, guanine-plus-cytosine content of deoxyribonucleic acid (DNA) from this organism (36.1 mol\% [31]) is distinctly lower than the range of values found in the genus Moraxella (4). In this study, the cellular. fatty acid composition of $\boldsymbol{H}$. equigenitalis was compared with the cellular fatty acid compositions of Moraxella species and species of other related genera to clarify possible relationships among these species.

\section{MATERIALS AND METHODS}

Bacterial strains and culture conditions. The strains used in this study are listed in Table 1 . The bacterial cells were cultivated on Eugon agar supplemented with $5 \%$ Fildes pepsin-digested sheep blood for $72 \mathrm{~h}$ under microaerophilic conditions. The cells were freeze-dried after they were washed three times with saline.

Extraction and methylation of fatty acids. The dried cells were treated with $5 \% \mathrm{HCl}$ in methanol at $105^{\circ} \mathrm{C}$ for $3 \mathrm{~h}$, and fatty acid methyl esters were extracted with hexane as previously described (21).

Determination of double bond positions in monounsaturated fatty acids. The monounsaturated fatty acid methyl esters in each mixture were converted to the corresponding dihydroxy fatty acids by oxidation with $\mathrm{OsO}_{4}$, as described by Capella and Zorzut (6). These dihydroxy fatty acids were subsequently converted to the corresponding ditrimethylsilyloxy ethers by reaction with hexamethyldisilazane and trimethylchlorosilane in pyridine (TMS-HT kit; Tokyo Chemical Industry Co. Ltd., Tokyo, Japan). The ditrimethylsilyloxy ethers of fatty acid methyl esters were extracted with chloroform and analyzed by gas chromatography-mass spectrometry (GC-MS). Double bond positions were determined by using $H$. equigenitalis NCTC 11184 and three Moraxella species (Moraxella bovis, Moraxella osloensis, and Moraxella urethralis).

GLC and GC-MS. Fatty acid methyl esters and the ditrimethylsilyloxy ethers of monounsaturated fatty acid methyl esters were analyzed by gas-liquid chromatography (GLC) and GC-MS as previously described (22). GLC was performed with a HewlettPackard model 5710A gas chromatograph equipped with a flexible fused-silica capillary column $(0.28 \mathrm{~mm}$ by $50 \mathrm{~m}$ ) coated with OV-101, GC-MS was performed by using a gas chromatograph-mass spectrometercomputer system (model 5992B; Hewlett-Packard) equipped with a glass capillary column $(0.28 \mathrm{~mm}$ by 30 $\mathrm{m})$ coated with OV-101. Fatty acid methyl esters were analyzed at an oven temperature of $180^{\circ} \mathrm{C}$, and the ditrimethylsilyloxy ethers were analyzed at $200^{\circ} \mathrm{C}$.

Identification of fatty acids. Fatty acids were identified primarily by equivalent chain length (ECL) calculations, using a standard mixture of fatty acid methyl esters, as previously described (22). The methyl esters of oleic acid ( $\Delta 918: 1$ acid) and cis-vaccenic acid ( $\Delta 11$ 18:1 acid) were also used as monounsaturated fatty acid standards. All structural determinations of fatty acids were verified by GC-MS.

Numerical analysis. The similarities among the strains tested based on cellular fatty acid composition 
TABLE 1. Strains used in this study

\begin{tabular}{|c|c|c|}
\hline $\begin{array}{c}\text { Serial } \\
\text { no. }\end{array}$ & Received as: & Source $^{a}$ \\
\hline 1 & M. atlantae ATCC $29525^{\mathrm{T} b}$ & 1 \\
\hline 2 & M. bovis ATCC $10900^{\mathrm{T}}$ & 1 \\
\hline 3 & M. catarrhalis ATCC $25238^{\mathrm{T}}$ & 1 \\
\hline 4 & $M$. caviae ATCC $14659^{\mathrm{T}}$ & 1 \\
\hline 5 & $M$. equi ATCC $25576^{\mathrm{T}}$ & 1 \\
\hline 6 & M. lacunata ATCC $17967^{\mathrm{T}}$ & 1 \\
\hline 7 & M. nonliquefaciens ATCC $19975^{\mathrm{T}}$ & 1 \\
\hline 8 & M. osloensis ATCC $19976^{\mathrm{T}}$ & 1 \\
\hline 9 & M. ovis ATCC $33078^{\mathrm{T}}$ & 1 \\
\hline 10 & M. phenylpyruvica ATCC $23333^{\mathrm{T}}$ & 1 \\
\hline 11 & $M$. urethralis ATCC $17960^{\mathrm{T}}$ & 1 \\
\hline 12 & M. anatipestifer ATCC $11845^{\mathrm{T}}$ & 1 \\
\hline 13 & M. anatipestifer NIH 80032 & 2 \\
\hline 14 & L. pneumophila ATCC 33154 & 1 \\
\hline 15 & L. pneumophila ATCC 33156 & 1 \\
\hline 16 & K. kingae ATCC $23330^{\mathrm{T}}$ & 1 \\
\hline 17 & H. equigenitalis NCTC 11184 & 3 \\
\hline 18 & H. equigenitalis Kentucky-188 & 4 \\
\hline 19 & H. equigenitalis EQ 51 & 5 \\
\hline 20 & H. equigenitalis EQ 56 & 5 \\
\hline 21 & H. equigenitalis $\mathrm{EQ} 59$ & 5 \\
\hline 22 & H. equigenitalis EQ 64 & 5 \\
\hline 23 & H. equigenitalis EQ 70 & 5 \\
\hline 24 & H. equigenitalis EQ CHIBA & 5 \\
\hline
\end{tabular}

${ }^{a}$ Sources: 1, American Type Culture Collection, Rockville, Md.; 2, R. Sakazaki, National Institute of Health, Tokyo, Japan; 3, National Collection of Type Cultures, London, England; 4, Y. Akiyama, Tochigi Branch Laboratory, Equine Health Laboratory, Tochigi, Japan (originally supplied by J. T. Bryans, College of Agriculture, University of Kentucky, Lexington); 5 , our collection.

b Type strain.

were calculated by using the formula of Bergan (2), as previously described (21).

\section{RESULTS}

The cellular fatty acid compositions of Moraxella, Kingella, and Legionella species and $H$. equigenitalis NCTC 11184 are shown in Table 2. Species belonging to the genus Moraxella generally contained large amounts of $16: 0$ and $18: 1$ acids, and the fatty acid compositions of these species were grossly similar to the fatty acid composition of $\boldsymbol{H}$. equigenitalis (30). Kingella kingae contained 14:0,16:1, and 16:0 acids as the major fatty acids and produced a fatty acid pattern that was different from the patterns of Moraxella species and $H$. equigenitalis. Moraxella anatipestifer and Legionella pneumophila contained large amounts of branched-chain fatty acids, especially iso-15:0 acid, and produced fatty acid patterns that were distinctly different from those of the other species tested.

The ECL values of the 18:1 acid indicated that positional isomers might exist. Because the methyl esters of positional isomers produce very similar mass spectra, the positions of double bonds could not be determined directly from the spectra. To determine the double bond positions, monounsaturated fatty acid methyl esters were converted to the corresponding ditrimethylsilyloxy derivatives and then analyzed by GCMS. GLC patterns of fatty acid methyl esters of $H$. equigenitalis before and after derivation are shown in Fig. 1 as examples. After derivation, the peak of the $16: 1$ acid completely disappeared, whereas a reduced amount of the 18:1 acid was still detected. Two new peaks, which appeared at retention times of about $\mathbf{1 7 . 5}$ and 33 min, were thought to correspond to the ditrimethylsilyloxy derivatives of 16:1 and 18:1 acids, respectively. The mass spectra of the ditrimethylsilyloxy derivatives of monounsaturated fatty acid methyl esters detected in $H$. equigenitalis and $M$. bovis, are shown in Fig. 2. These spectra show a characteristic cleavage between two trimethylsilyloxy groups, resulting in very simple mass spectra that are dominated by the two cleavage fragments, as shown in Fig. 3 (6). The peak at $m / e$ 73 was due to the trimethylsilyl ion. $H$. equigenitalis and $M$. urethralis contained $\Delta 11 \quad 18: 1$ acid and palmitoleic acid $(\Delta 9$ 16:1 acid), and $H$. equigenitalis also contained a trace of $\Delta 918: 1$ acid. $M$. bovis and $M$. osloensis contained $\Delta 918: 1$ and $\Delta 916: 1$ acids, and $\Delta 917: 1$ acid was detected in $M$. bovis. The double bond positions of 18:1 acids detected in the other species, which were not included in our determination of double bond positions by trimethylsilyloxy derivation, were easily deduced from their ECL values. The ECL value of $\Delta 1118: 1$ acid was $17.77 \pm 0.01$, and that of $\Delta 918: 1$ acid was $17.71 \pm 0.01$.

After we calculated the correlation coefficient for all of the strains, we constructed a dendrogram based on cellular fatty acid composition (Fig. 4). Moraxella species formed two clusters. The first cluster included Moraxella catarrhalis, Moraxella ovis, and $M$. urethralis, which contained cis-vaccenic acid as a major fatty acid. The strains of $\boldsymbol{H}$. equigenitalis were very similar to each other and were closely related to the cisvaccenic acid group of Moraxella species. The other Moraxella species (Moraxella atlantae, M. bovis, Moraxella caviae, Moraxella equi, Moraxella lacunata, Moraxella nonliquefaciens, M. osloensis, and Moraxella phenylpyruvica) formed the oleic acid group. $M$. anatipestifer and $L$. pneumophila were separated from the two clusters of Moraxella species. K. kingae could be distinguished from the Moraxella species by cellular fatty acid composition.

\section{DISCUSSION}

We previously studied the cellular fatty acid composition of $H$. equigenitalis (30) and found 


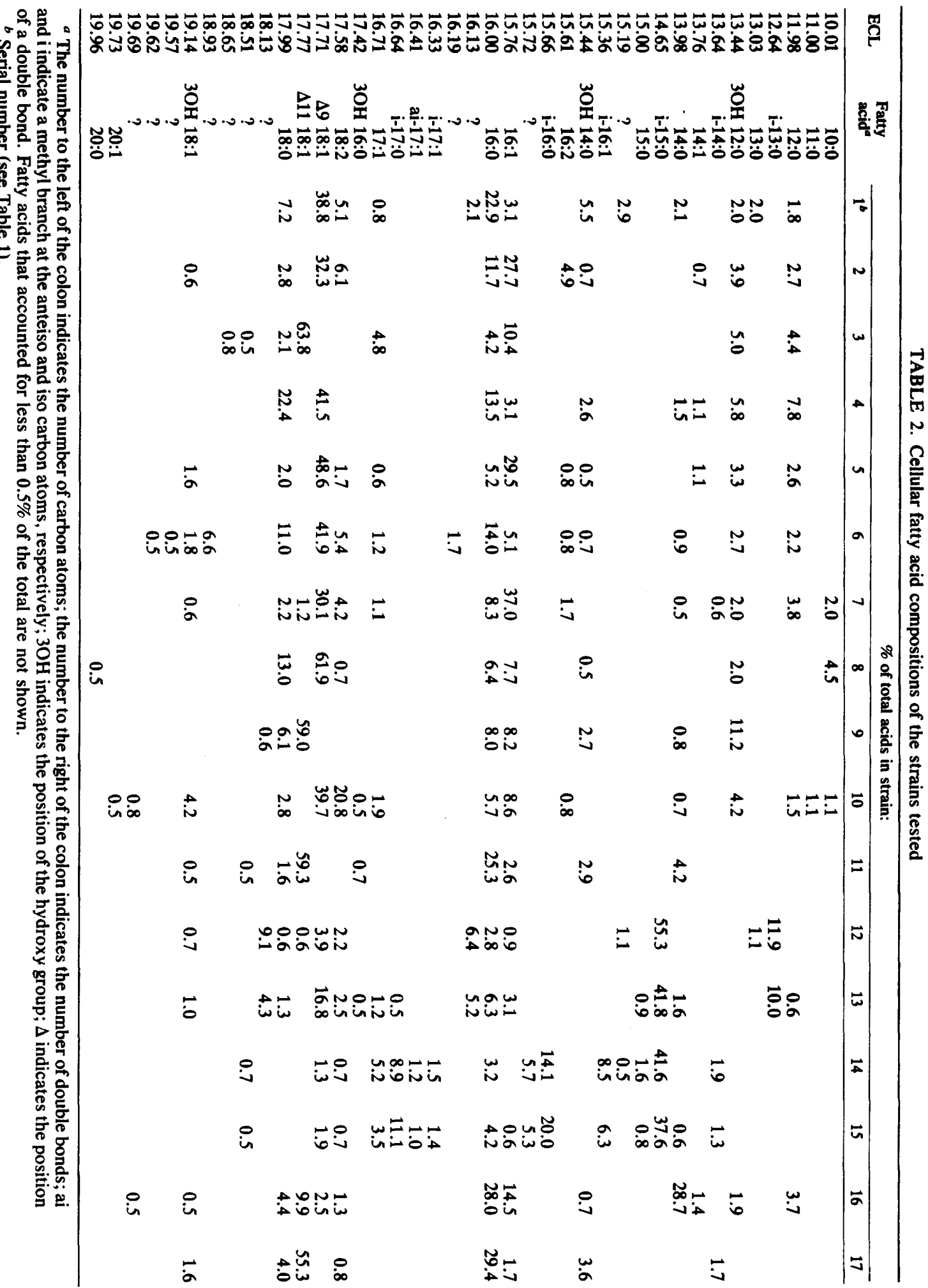



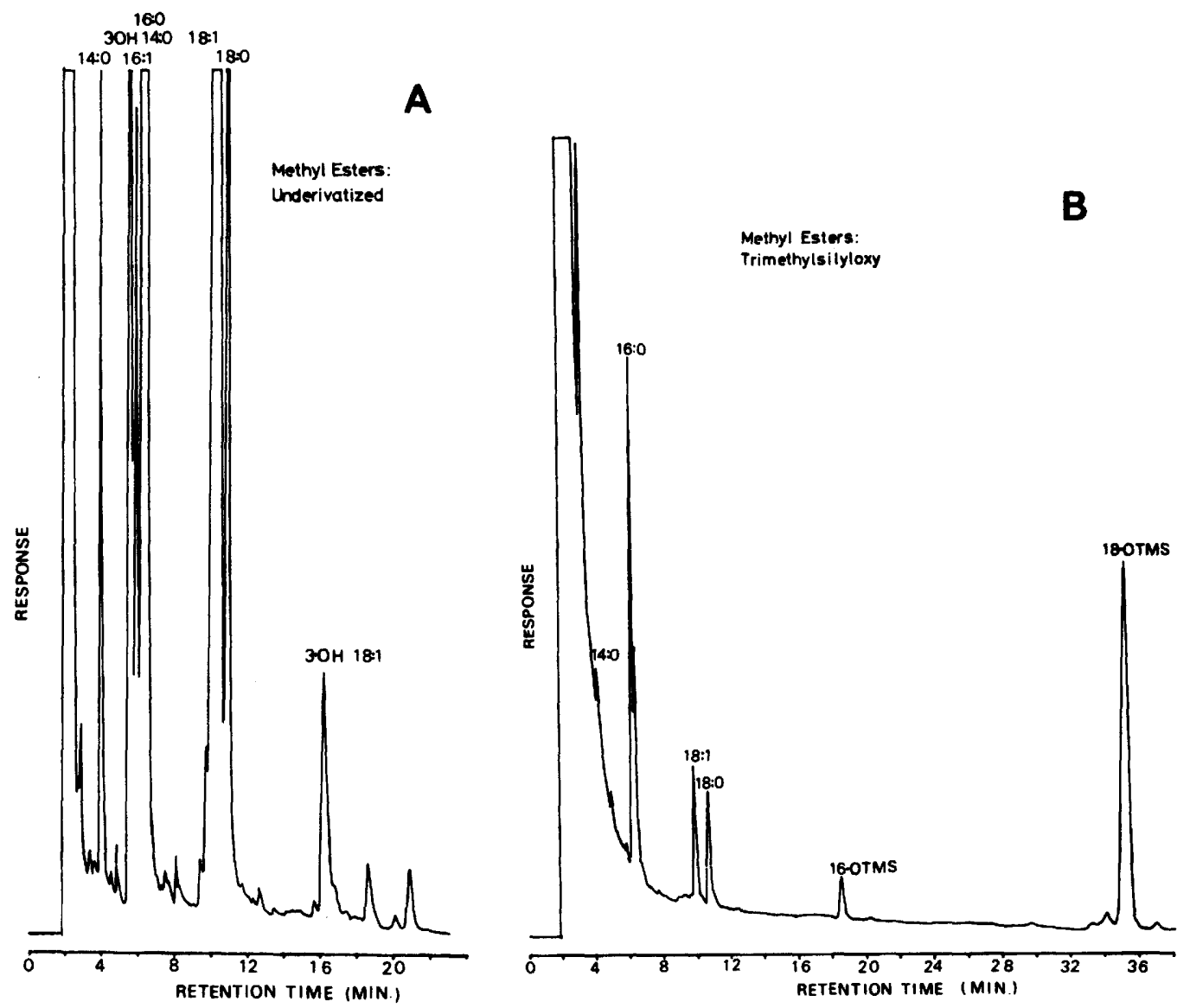

FIG. 1. Gas chromatograms of fatty acid methyl esters of $H$. equigenitalis NCTC 11184 before (A) and after (B) ditrimethylsilyloxy derivation. The analysis was done on an OV-101 column at an oven temperature of $200^{\circ} \mathrm{C}$; 16-OTMS and 18-OTMS indicate the ditrimethylsilyloxy derivatives of 16:1 and 18:1 acids, respectively.

that it is clearly distinct from the cellular fatty acid compositions of Haemophilus species (13) and grossly similar to the cellular fatty acid compositions of Moraxella species $(5,9,14,15$, 27). Moraxella species have been reported to contain large amounts of 16:0 and 18:1 acids, which we confirmed in this study. The cellular fatty acid composition of $M$. anatipestifer, whose biochemical characteristics are markedly different from those of other Moraxella species (1), was peculiar in its abundance of branchedchain fatty acids and was similar to the cellular fatty acid compositions of Legionella species $(23,24,26)$.

B $\emptyset$ vre and colleagues studied the cellular fatty acid compositions of Moraxella and Neisseria species $(5,9,14,15)$ and found that false Neisseria or Branhamella (i.e., $M$. catarrhalis, $M$. caviae, and $M$. ovis) strains have fatty acid patterns grossly similar to those of Moraxella species. This observation is one of the reasons that the false Neisseria species were reclassified in the genus Moraxella. Bøvre described the taxonomic problems encountered with the false Neisseria species and proposed to divide the genus Moraxella into the following two subgenera, based mainly on cell shape: subgenus Moraxella, containing rod-shaped organisms, and subgenus Branhamella, containing cocci (3).

However, B $\varnothing v r e$ et al. did not distinguish positional isomers of 18:1 acid, which is a major fatty acid in Moraxella species. Two species of subgenus Branhamella, $M$. catarrhalis and $M$. ovis, were clearly separated from species belonging to subgenus Moraxella and $M$. caviae by the positional isomer compositions of 18:1 acids in the present study. The positional isomers of 18:1 acid have taxonomic significance in the genus Moraxella since this genus has been divided into the oleic acid group and the cis-vaccenic acid group. The double bond positions in 18:1 acids were easily deduced from ECL values when a GLC analysis was performed by using a flexible fused capillary column because of its 


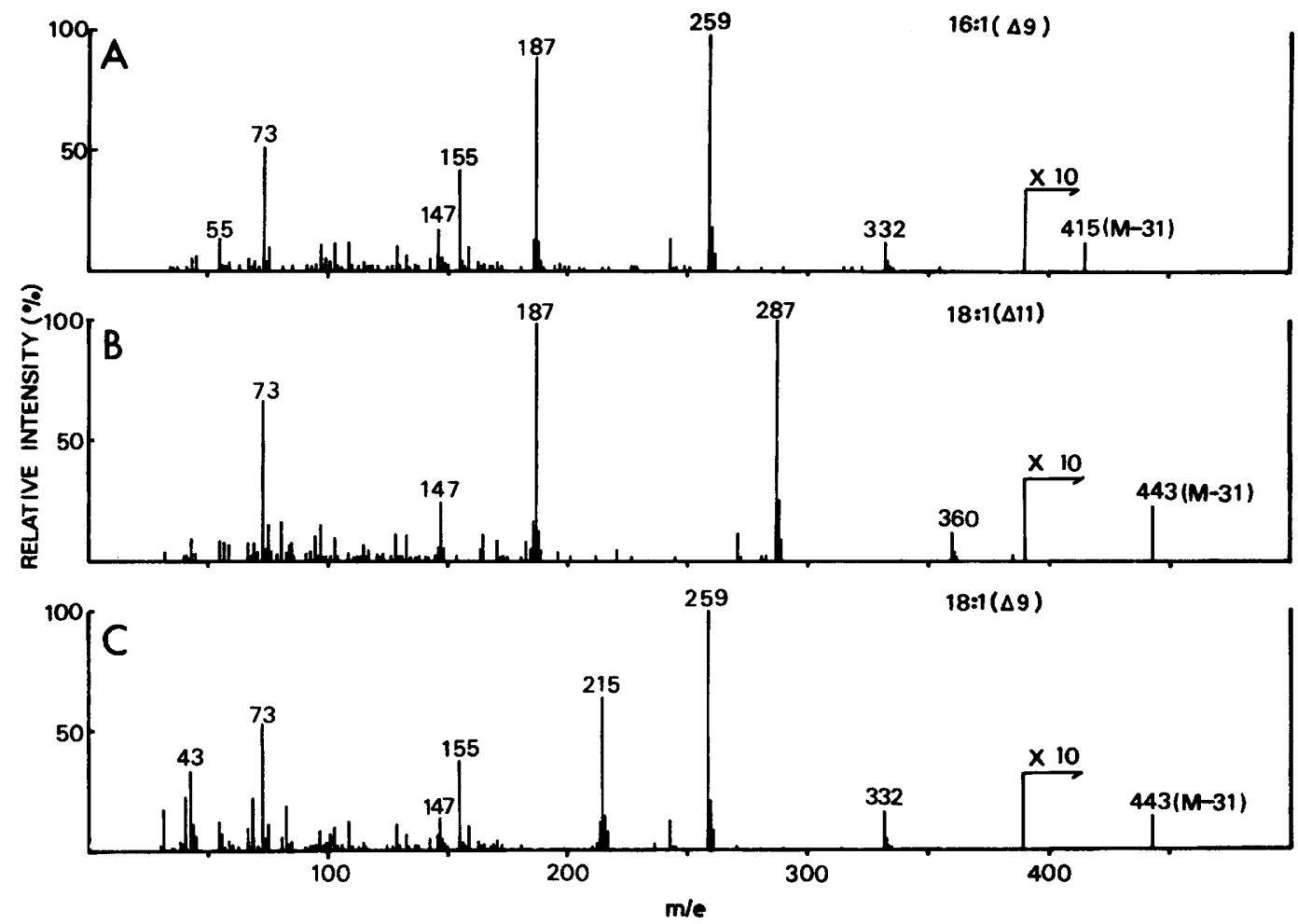

FIG. 2. Mass spectra of ditrimethylsilyloxy derivatives of monounsaturated fatty acid methyl esters. $\Delta 916: 1$ acid (A) and $\Delta 11$ 18:1 acid (B) were detected in $H$. equigenitalis, and $\Delta 918: 1$ acid (C) was detected in $M$. bovis.

high resolution and separation capacity, as reported by Moss et al. (25).

In contrast to the previous finding that $M$. urethralis is separated from other Moraxella species by cellular fatty acid composition (14, 15), $M$. urethralis, $M$. catarrhalis, and $M$. ovis clustered together in our study. This apparently resulted from differences in the methods used to calculate similarity. The primary logarithmic transformation of the data for each fatty acid used in the previous studies may have given relatively more weight to fatty acids that occur in small quantities. For example, $\boldsymbol{M}$. urethralis contained relatively small amounts of $14: 0$ and

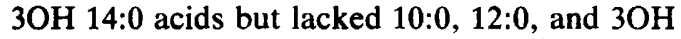
16:0 acids, in contrast to $M$. catarrhalis and $M$. ovis. Furthermore, the amount of $16: 1$ acid was relatively smaller than the amount of $16: 0$ acid in $M$. urethralis compared with the other two species.

It is known that unsaturated fatty acids are synthesized from saturated fatty acids by two pathways (aerobic and anaerobic) $(8,10,11)$. The anaerobic pathway of desaturation generally leads to palmitoleic and cis-vaccenic acids as the major products, whereas the aerobic pathway yields primarily palmitoleic and oleic acids
(10). The detection of $\Delta 9$ monounsaturated fatty acids in $M$. bovis and $M$. osloensis strongly suggests that these organisms have the aerobic pathway of desaturation at the $\Delta 9$ position. The existence of this pathway in gram-negative bacteria is known only in Alcaligenes faecalis, although it is common in gram-positive bacteria (17). On the other hand, the detection of $\omega 7$ monounsaturated fatty acids in $\boldsymbol{H}$. equigenitalis and $M$. urethralis indicates the existence of the anerobic pathway of desaturation. $H$. equigenitalis synthesizes a trace amount of oleic acid, probably by the anaerobic pathway since the two pathways are mutually exclusive in the sense that no organism which possesses both

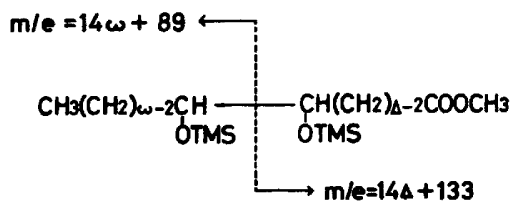

FIG. 3. Typical cleavage of ditrimethylsilyloxy derivatives (OTMS) of a monounsaturated fatty acid methyl ester. $\Delta$ and $\omega$ indicate the numbers of carbon atoms measured from the terminal methyl base and carboxyl base, respectively. 


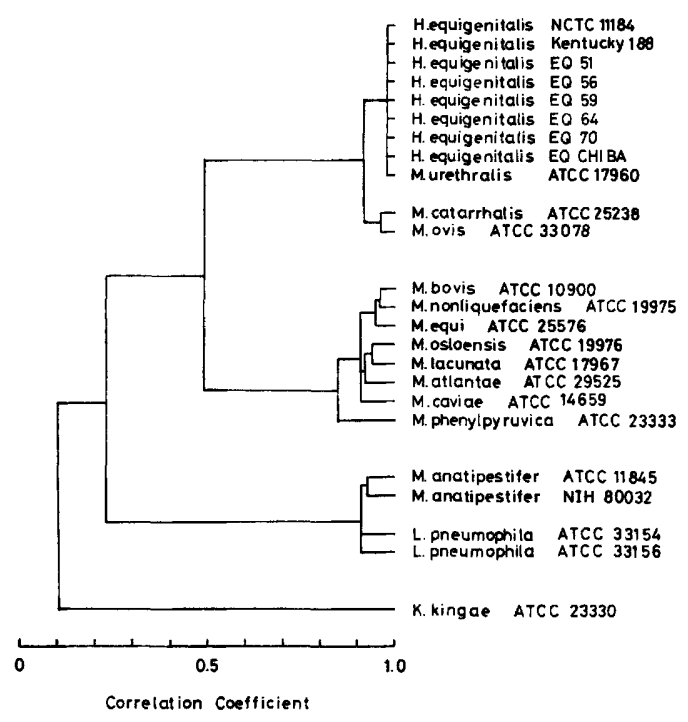

FIG. 4. Dendrogram of the strains tested based on cellular fatty acid composition. The correlation coefficient was calculated by the formula of Bergan (2).

pathways has been found $(8,11)$.

In our previous study of $\boldsymbol{H}$. equigenitalis (30), we found no significant difference in cellular fatty acid composition among cells cultivated on different media even if we substituted a chemically defined growth supplement, such as IsoVitaleX (BBL Microbiology Systems, Cockeysville, Md.), for blood components. However, we cannot rule out the possibility that some species tested in this study incorporate fatty acids from the medium, as indicated by Letts et al. (20). If this is the case, the differences in cellular fatty acid composition result from differences in the ability to incorporate lipid constituents from the medium rather than differences in pathways of desaturation. Because only a few species contain both of oleic and cis-vaccenic acids and either of these acids is dominant in amount, the possibility that the dominant positional isomer was incorporated from the medium is unlikely. However, the pathway by which organisms synthesize monounsaturated fatty acids must be proved directly by using labeled precursors.

Taylor et al. proposed that the causative organism of contagious equine metritis should be classified as a member of the genus Haemophilus based on the guanine-plus-cytosine content of its DNA and its X-factor requirement, and these authors designated this organism $H$. equigenitalis (31). The X-factor requirement of this organism was reported by Shreeve (28), but Taylor et al. (31), Dabernat et al. (7), and we could not confirm this result. Because the porphyrin test for this organism is positive, like other X-factor-independent Haemophilus spe- cies (16), this organism does not require $X$ factor. $H$. equigenitalis is a catalase- and oxidase-positive asaccharolytic coccobacillus, is very unreactive in conventional biochemical tests, and shows phenotypic similarity to Moraxella and Legionella species. In cellular fatty acid composition, $H$. equigenitalis is very similar to the cis-vaccenic acid group of Moraxella species but distinct from the oleic acid group of Moraxella species and Legionella species.

When other taxonomic criteria are taken in consideration, $H$. equigenitalis differs from the Moraxella species described above in some characteristics. The guanine-plus-cytosine contents of $M$. urethralis, $M$. catarrhalis, $M$. ovis, and $H$. equigenitalis DNAs are 46 to $47,46.5$ to $47.5,44.5$ to 46.5 , and $36.1 \mathrm{~mol} \%$, respectively $(4,31)$. Furthermore, $M$. urethralis, which has been tentatively placed in the genus Moraxella despite genetic incompatibility with this genus, grows slowly in a simple mineral medium supplemented with ammonium ions as the source of nitrogen and acetate or hydroxybutylate as the carbon source (19). The nutritional properties of $H$. equigenitalis, which have not been reported, seem rather complex.

Further taxonomic studies based on DNADNA hybridizations and cellular enzyme profiles are now in progress to clarify the possible relationship of $\boldsymbol{H}$. equigenitalis to the family Neisseriaceae.

\section{ACKNOWLEDGMENT}

We thank R. Sakazaki, National Institute of Health, Tokyo, Japan, for his advice and stimulating discussions.

\section{LITERATURE CITED}

1. Bangun, A., D. N. Tripathy, and L. E. Hanson. 1980. Studies on Pasteurella anatipestifer: an approach to its classification. Avian Dis. 25:326-336.

2. Bergan, T. 1972. Survey of numerical techniques for grouping. Bacteriol. Rev. 35:379-389.

3. Bøvre, K. 1979. Proposal to divide the genus Moraxella Lwoff 1939 emend. Henriksen and Bøvre 1968 into two subgenera, subgenus Moraxella (Lwoff 1939) Bøvre 1979 and subgenus Branhamella (Catlin 1970) Bøvre 1979. Int. J. Syst. Bacteriol. 29:403-406.

4. Bøvre, K., and N. Hagen. 1981. The family Neisseriaceae: rod-shaped species of the genera Moraxella, Acinetobacter, Kingella, and the Branhamella group of cocci, p. 1506-1529. In M. P. Starr, H. Stolp, H. G. Trüper, A. Balow, and H. G. Schlegel (ed.), The prokaryotes. Springer-Verlag, Berlin.

5. Bøvre, K., R. Hytta, E. Jantzen, and L. O. Frøholm. 1972. Gas chromatography of bacterial whole cell methanolysates. III. Group relations of Neisseriae and Moraxellae. Acta Pathol. Microbiol. Scand Sect. B 80:683-689.

6. Capella, P., and C. M. Zorzut. 1968. Determination of double bond position in monounsaturated fatty acid esters by mass spectrometry of their trimethylsilyloxy derivatives. Anal. Chem. 40:1458-1463.

7. Dabernat, H. J., D. Tainturier, C. Delmas, J. Ferney, and M. B. Lareng. 1980. Étude bactériologique de Haemophilus equigenitalis Taylor 1978, agent de la métrite contagieuse de la jument. Ann. Rech. Vet. 11:289-299.

8. Edwin, J., and K. Bloch. 1964. Biosynthesis of unsaturated fatty acids in microorganisms. Science 143:1006-1012. 
9. Frøholm, L. O., E. Jantzen, R. Hytta, and K. Bøvre. 1972. Gas chromatography of bacterial whole cell methanolysates. II. A taxonomic evaluation of the method for species of Moraxella. Acta Pathol. Microbiol. Scand. Sect. B 80:672-682.

10. Fulco, A. J. 1974. Metabolic alterations of fatty acids. Annu. Rev. Biochem. 43:215-241.

11. Gurr, M. I. 1974. The biosynthesis of unsaturated fatty acids, p. 181-235. In T. W. Goodwin (ed.), Biochemistry of lipids. Biochemistry series 1 , vol. 4. Butterworth, London.

12. Henriksen, S. D. 1976. Moraxella, Neisseria, Branhamella, and Acinetobacter. Annu. Rev. Microbiol. 30:6383.

13. Jantzen, E., B. P. Berdal, and T. Omland. 1980. Cellular fatty acid composition of Haemophilus species, Pasteurella multocida, Actinobacillus actinomycetemcomitans and Haemophilus vaginalis (Corynebacterium vaginale). Acta Pathol. Microbiol. Scand. Sect. B 88:89-93.

14. Jantzen, E., K. Bryn, T. Bergan, and K. Bøvre. 1974. Gas chromatography of bacterial whole cell methanolysates. V. Fatty acid composition of Neisseriae and Moraxellae. Acta Pathol. Microbiol. Scand. Sect. B 82:767-779.

15. Jantzen, E., K. Bryn, T. Bergan, and K. Bøvre. 1975. Gas chromatography of bacterial whole cell methanolysates. VII. Fatty acid composition of Acinetobacter in relation to the taxonomy of Neisseriaceae. Acta Pathol. Microbiol. Scand. Sect. B 83:569-580.

16. Kilian, M. 1976. A taxonomic study of the genus Haemophilus, with the proposal of a new species. J. Gen. Microbiol. 93:9-62.

17. Kshirsagar, S. S., and P. Nair. 1979. Isolation and properties of a particulate fraction for the desaturation of palmitic acid from Alcaligenes faecalis. Biochim. Biophys. Acta 574:369-378.

18. Lambert, M. A., D. G. Hollis, C. W. Moss, R. E. Weaver, and M. L. Thomas. 1971. Cellular fatty acids of nonpathogenic Neisseria. Can. J. Microbiol. 17:1491-1502.

19. Lautrop, H., K. Bøvre, and W. Frederiksen. 1970. A Moraxella-like microorganism isolated from the genitourinary tract of man. Acta Pathol. Microbiol. Scand. Sect. B 78:255-256.
20. Letts, V., P. Shaw, L. Shapiro, and S. Henry. 1982. Synthesis and utilization of fatty acids by wild-type and fatty acid auxotrophs of Caulobacter crescentus. J. Bacteriol. 151:1269-1278.

21. Miyagawa, E., R. Axuma, and T. Suto. 1979. Cellular fatty acid composition in gram-negative obligately anaerobic rods. J. Gen. Appl. Microbiol. 25:41-51.

22. Miyagawa, E., and T. Suto. 1980. Cellular fatty acid composition in Bacteroides oralis and Bacteroides ruminicola. J. Gen. Appl. Microbiol. 26:331-343.

23. Moss, C. W. 1981. Gas-liquid chromatography as an analytical tool in microbiology. J. Chromatogr. 203:337-347.

24. Moss, C. W., and S. B. Dees. 1979. Cellular fatty acid composition of WIGA, a rickettsia-like agent similar to the Legionaires disease bacterium. J. Clin. Microbiol. 10:390-391.

25. Moss, C. W., Shinoda, T., and J. W. Samuels. 1982. Determination of cellular fatty acid compositions of various yeasts by gas-liquid chromatography. J. Clin. Microbiol. 16:1073-1079.

26. Moss, C. W., R. E. Weaver, S. B. Dees, and W. B. Cherry. 1977. Cellular fatty acid composition of isolates from Legionaires disease. J. Clin. Microbiol. 6:140-143.

27. Nishimura, Y., H. Yamamoto, and H. lizuka. 1979. Taxonomical studies of Acinetobacter species. Cellular fatty acid composition. Z. Alig. Mikrobiol. 19:307-308.

28. Shreeve, J. E. 1978. Preliminary observations on X-factor requirement of the bacterium responsible for CEM. Vet. Rec. 102:20.

29. Sugimoto, C., Y. Isayama, M. Kashiwazaki, T. Fujikura, and K. Mitani. 1980. Detection of Haemophilus equigenitalis, the causal agent of contagious equine metritis, in Japan. NatI. Inst. Anim. Health Q. 20:118-119.

30. Sugimoto, C., E. Miyagawa, K. Mitani, M. Nakazawa, and Y. Isayama. 1982. Cellular fatty acid composition of Haemophilus equigenitalis. J. Clin. Microbiol. 15:791794.

31. Taylor, C. E. D., R. O. Rosenthal, D. F. J. Brown, E. S. P. Lapage, L. R. Hill, and R. M. Legros. 1978. The causative organism of contagious equine metritis 1977 proposal for a new species to be known as Haemophilus equigenitalis. Equine Vet. J. 10:136-144. 